ISSN 0103-5150

Fisioter. Mov., Curitiba, v. 25, n. 2, p. 361-368, abr./jun. 2012 Licenciado sob uma Licença Creative Commons

\title{
Influência do peso adicional nos chutes de lactentes pré-termo e de termo
}

\author{
Influence of additional weight on kicks of \\ preterm and full-term infants
}

\section{Jadiane Dionisio $^{[\mathrm{a}]}$, Gabriela Lopes dos $\operatorname{Santos}^{[\mathrm{b}]}$, Jocelene de Fátima Landgraf ${ }^{[\mathrm{c}]}$, Eloisa Tudella ${ }^{[\mathrm{d}]}$}

[a] Fisioterapeuta, Mestre pela Universidade Federal de São Carlos (UFSCar), São Carlos, SP - Brasil, e-mail: jadydionisio@hotmail.com

[b] Acadêmica de Fisioterapia pela Universidade Federal de São Carlos (UFSCar), São Carlos, SP - Brasil, e-mail: gabrielalsantos@hotmail.com

[c] Fisioterapeuta, Doutora pela Universidade Federal de São Carlos (UFSCar), São Carlos, SP - Brasil e docente do curso de fisioterapia da Universidade Federal do Rio de Janeiro (UFRJ), Rio de Janeiro - RJ, Brasil, e-mail: jolandgraf@gmail.com

[d] Doutora em Psicologia pela Universidade de São Paulo (USP), São Paulo - Brasil, docente associada nível III do curso de Fisioterapia e coordenadora do Núcleo de Estudos em Neuropediatria e Motricidade da Universidade Federal de São Carlos (UFSCar), São Carlos, SP - Brasil, e-mail: eloisatudella@yahoo.com

\section{Resumo}

Introdução: O objetivo do estudo foi analisar e comparar padrões de chutes com e sem peso adicional em lactentes pré-termo e de termo aos 3 e 4 meses de idade. Materiais e métodos: Foram filmados 26 lactentes aos 3 e 4 meses de idade, divididos em Grupo Experimental, 12 lactentes pré-termo sem idade corrigida; e Grupo Controle, 14 lactentes de termo. 0 experimento foi subdividido em quatro condições, a saber: 1) Treinamento (T): o pesquisador tocava os pés dos lactentes no painel, elevando-o; 2) Linha de Base (LB): movimentos livres de chutes, podendo o lactente elevar ou não o painel com os pés; 3) Peso (P): acréscimo de tornozeleira pesando $1 / 3$ da massa do membro inferior; 4) Pós-Peso (PP): idêntica à LB. Resultados: Na variável frequência de chutes, constatou-se diferença entre as idades $[F(5,72)=5,620 ; p=0,0035]$ e entre as condições LB-P ( $p=0,023)$ e P-PP ( $<<0,001)$. Na variável frequência de contato, obteve-se diferença entre as condições LB-P ( $p=0,002)$ e P-PP ( $<<0,001)$. Na variável sucesso ao elevar o painel, constatou-se diferença entre os grupos $\left(x^{2}{ }_{(1)}=4,341 ; p=0,0013\right)$ e as condições LB-P $(p=0,003)$, LB-PP $(p=0,002)$ e P-PP $(\mathrm{p}=0,002)$ aos 3 meses de idade. Na variável duração de chute, constatou-se diferença entre as condições 
LB-P ( $p=0,023)$ e P-PP ( $p=0,010)$. Conclusão: Os chutes de lactentes pré-termo se diferenciam dos termos dependendo das características intrínsecas e extrínsecas.

Palavras-chave: Desenvolvimento. Chutes. Peso adicional. Pré-termo. Lactentes.

\section{Abstract}

Introduction: The aim of this study was to analyze and compare patterns of kicks with and without additional weight in preterm and full-term infants of 3 and 4 months old. Materials and methods: Twenty-six infants were filmed at 3 and 4 months of age, divided into experimental group, 12 preterm infants without corrected age, and control group, 14 infants full-term. The experiment was subdivided into four conditions, namely: 1) Training (T): the investigator touched the infants' feet on the panel, raising it; 2) Baseline (B): free movements of kicks, the infant may raise or not the panel with feet, 3) Weight (W): addition of anklet weighing 1/3 the mass of lower limb, 4) Post-weight (PW): identical to B. Results: Difference was found in the variable frequency of kicks, considering ages $[F(5,72)=5.620, p=0.0035]$ and conditions $B-W(p=0.023)$ and $W$-PW $(p<0.001)$. In the variable frequency of contact difference was obtained considering conditions $B-W$ $(p=0.002)$ and $W-P W(p<0.001)$. In the variable success in raising the panel, difference was found between groups $\left(x_{(1)}^{2}=4.341, p=0.0013\right)$ and conditions $B-W(p=0.003), B-W(p=0.002)$ and $W-P W(p=0.002)$ at 3 months of age. The variable length of the kick presented a difference between conditions $B-W(p=0.023)$ and $W-P W(p=0.010)$. Conclusion: The kicks of preterm infants differ depending on the intrinsic and extrinsic characteristics.

Keywords: Development. Kicks. Additional weight. Preterm. Infant.

\section{Introdução}

O recém-nascido pré-termo pode apresentar atraso no desenvolvimento neuromotor e imaturidade funcional e estrutural dos sistemas em função do menor tempo de vida intrauterina (1), o que o torna um dos maiores grupos de risco para alterações e atrasos motores (2-7).

Em virtude da imaturidade funcional e estrutural, o recém-nascido pré-termo apresenta hipotonia generalizada e padrão flexor diminuído, o que favorece a postura extensora e altera a ativação do tônus muscular, semelhante ao do lactente de termo. Além desses fatores, o lactente pré-termo pode apresentar movimentos espontâneos lentos de membros inferiores e superiores, ocasionando atraso na aquisição das habilidades motoras futuras (8). Este quadro pode ser ainda mais prejudicado em razão da força da gravidade, que atua contra a musculatura fraca, dificultando a aquisição de marcos motores antigravitacionais, pois exige um aumento pronunciado do tônus muscular, 0 que impede a realização de movimentos organizados (8-11). Essas características podem variar de acordo com a classificação do lactente pré-termo, sendo que, em lactentes muito prematuros e prematuros extremos (aqueles nascidos com idade gestacional de $33^{6 / 7}$ a $30^{6 / 7}$ e menor que 30 semanas, respectivamente), essas alterações são significativamente mais observadas.

Uma das habilidades motoras antigravitacionais primitivas que pode ser adquirida tardiamente pelo lactente pré-termo é o chute (12-15). A sua aquisição favorece o ganho de força e a coordenação dos membros inferiores, o que, por sua vez, parece ser imprescindível para a realização de habilidades mais complexas, como engatinhar, escalar e andar $(9,15,16)$.

0 desenvolvimento do chute implica a aquisição de movimentos em-fase e fora-de-fase, bem como de coordenações intra e intermembros, o que ocorre nos primeiros 10 meses de idade $(9,12-16)$. 0 desenvolvimento das coordenações intra e intermembros dos lactentes pré-termo ocorrem tardiamente quando comparado ao dos lactentes de termo nas idades corrigidas de 4, 8, 12, 16, 20 e 24 semanas (17).

0 chute é uma habilidade motora que, assim como outras, sofre influência da interação entre elementos do organismo, do ambiente e da especificidade da tarefa. Alguns autores realizaram estudo para verificar a influência de fatores extrínsecos ao organismo adicionando um peso de $50 \%$ da massa do membro 
inferior, em um dos membros inferiores de lactentes de termo com 6 semanas de idade. Eles observaram que a amplitude, a velocidade e a frequência dos chutes do membro com peso adicional foi mantida, enquanto a perna contralateral apresentou aumento dessas variáveis (18). Em estudo similar, outro autor constatou que lactentes de termo que apresentaram melhor performance do chute (amplitude e frequência) quando colocado o peso adicional adquiriram as funções motoras de engatinhar e caminhar precocemente e realizaram-nas com melhor desempenho (19).

Para verificar o desenvolvimento dos chutes de lactentes pré-termo, autores compararam as variáveis amplitude, frequência, velocidade e coordenação intramembro dos chutes de lactentes pré-termo com idade corrigida em quatro diferentes faixas etárias. Esses autores constataram que lactentes nas faixas etárias menores (6 e 12 semanas) apresentam diminuição nas variáveis quando comparados aos lactentes de faixas etárias maiores (18 e 26 semanas), reforçando a ideia de que o chute de forma madura ocorre tardiamente nos lactentes pré-termo (20).

Embora já tenha sido demonstrado que o acréscimo de peso interfere no comportamento do chute, poucos estudos investigaram tal influência em lactentes pré-termo. A lacuna existente na literatura justifica esse trabalho, onde os resultados fornecerão subsídios para melhor entendimento do desenvolvimento motor desses lactentes. Portanto, o presente estudo tem como finalidade analisar e comparar padrões de chutes com e sem peso adicional em lactentes pré-termo e de termo aos 3 e 4 meses de idade.

Para tanto, três hipóteses foram testadas:

1) As variáveis frequência dos chutes, frequência de contato dos pés no painel, frequência de sucesso de elevar o painel e duração do chute de lactentes pré-termo serão menores quando comparadas às de lactentes de termo em todas as idades e condições experimentais;

2) As variáveis frequência de chutes e frequência de contato serão maiores e as variáveis frequência de sucesso de elevar o painel e duração do chute serão menores aos 3 meses quando comparadas aos 4 meses para ambos os grupos;

3) Durante a condição experimental peso, as variáveis frequência de chutes e frequência de contato serão maiores e as variáveis frequên- cia de sucesso de elevar o painel e duração do chute serão menores quando comparadas às condições experimentais Linha de Base e Pós-Peso nos dois grupos aos 3 e 4 meses de idade.

\section{Materiais e métodos}

Este estudo de caráter longitudinal foi aprovado pelo Comitê de Ética e Pesquisa com Seres Humanos da Universidade Federal de São Carlos (parecer n. 081/2006).

\section{Participantes}

Participaram do estudo 26 lactentes saudáveis, de ambos os sexos, com 3 e 4 meses de idade, subdivididos em dois grupos: experimental, 12 lactentes pré-termo com idade gestacional média de 33,23 semanas $( \pm 3,13)$, peso médio ao nascimento de 2343,84 gramas $( \pm 446,53)$, comprimento médio ao nascimento de $42,731( \pm 0,65)$ e índice de Ápgar médio de $8,52( \pm 0,51)$ no primeiro minuto e 9,2 $( \pm 0,33)$ no quinto minuto; e controle, 14 lactantes de termo com idade gestacional média de 38,75 semanas $( \pm 0,86)$, peso médio ao nascimento de 3226,5 gramas $( \pm 465,51)$ e comprimento médio ao nascimento de 47,91 $( \pm 0,76)$ e índice de Ápgar médio de $8,86( \pm 0,71)$ e $9,5( \pm 0,52)$ no primeiro e quinto minuto, respectivamente.

Ressalta-se que o grupo experimental era composto por nove lactentes classificados como pré-termo tardios (IG: 34 a $36^{6 / 7}$ ) e três lactentes como muito prematuro (IG: 33 a $31^{6 / 7}$ ). Apesar de pertencerem a grupos distintos, estes foram homogeneizados em um único grupo, pois foram considerados adequados para idade gestacional (AIG) e apresentaram condições clínicas semelhantes.

A amostra foi calculada considerando-se um desvio padrão de $5 \mathrm{~g} / \mathrm{cm}^{2}$, erro amostral previsto de $2 \mathrm{~g} /$ $\mathrm{cm}^{2}$ e nível de significância de 5\% (21). 0 tamanho mínimo estimado da amostra foi de 20 recém-nascidos.

Foram excluídos do estudo os lactentes com malformações do SNC, síndromes genéticas, deficiências sensoriais (auditiva e visual), cognitivas, cardiorrespiratórias ou ortopédicas; ou qualquer outra alteração do desenvolvimento neuro-sensório-motor, cognitivo e afetivo. Os desligamentos dos lactentes do estudo ocorreram quando faltaram a qualquer uma das 
duas avaliações; quando os responsáveis desistiram de participar do estudo; ou quando apresentaram intercorrências que poderiam prejudicar os resultados da pesquisa.

\section{Procedimentos}

Os sujeitos foram selecionados em uma Unidade Básica de Saúde da cidade de São Carlos e os pais dos lactentes assinaram o Termo de Consentimento Livre e Esclarecido, autorizando a participação de seus filhos no estudo. Os lactentes foram avaliados na data de aniversário, considerando um intervalo de, aproximadamente, cinco dias.

No laboratório, os lactentes foram despidos pela mãe e o examinador coletou suas medidas antropométricas (peso e comprimento total do corpo, comprimento e circunferência das coxas e pernas, e comprimento e largura dos pés) para estimar a massa de cada membro inferior (22).

Após esses procedimentos, os lactentes eram posicionados em supino em uma mesa desenvolvida para o estudo e estabilizados pelos ombros por um auxiliar de pesquisa. Na extremidade inferior da cadeira havia um painel que, ao ser elevado pelos pés do lactente, acionava um móbile fixado a uma altura de, aproximadamente, 30 centímetros acima do rosto do mesmo, agindo como estímulo visual e auditivo para a realização dos chutes (23). A posição do painel foi definida pela multiplicação do comprimento do membro inferior do lactente pelo seno $30^{\circ}(24)$.

0 experimento teve duração total de cerca de cinco minutos, subdivididos em quatro condições: 1) Treinamento (T): os pés dos lactentes foram colocados pelo examinador no painel para ativar o móbile, atividade realizada três vezes com cada membro separadamente e três vezes com os dois membros juntos, por três segundos cada; 2) Linha de Base (LB): os lactentes ficavam livres para realizar os chutes, podendo elevar o painel com os pés e acionar o móbile; 3) Peso (P): idêntica à condição anterior, porém, colocava-se nos tornozelos do lactente uma tornozeleira confeccionada de tecido com chumbinhos, que eram adicionados até chegar ao peso $1 / 3$ da massa do membro inferior do referido lactente; 4) Pós-Peso (PP): idêntica à LB. Em cada condição, admitiu-se o tempo de um (1) minuto para a realização dos chutes, e entre as condições 2, 3 e 4 havia um intervalo de 30 segundos para a colocação ou retirada das tornozeleiras. Em todo o procedimento, os lactentes permaneceram em estado de alerta inativo ou ativo (25).

Para registro das avaliações foram utilizadas duas câmeras digitais JVC (modelo GY DV-300) acopladas a tripés posicionados anterolateralmente à cadeira, uma em cada lado, e dois iluminadores posicionados ao lado das câmeras e direcionados para a parede para não prejudicar a visualização da imagem capturada.

\section{Variáveis analisadas}

Chute foi definido como movimento de um ou ambos os membros inferiores dos lactentes, partindo da flexão total de quadril, joelho e tornozelo para extensão e retornando para a posição inicial, indicando, assim, um ciclo de chute.

A partir dessa definição, os chutes foram categorizados em: 1) com contato, os chutes que seguiram em direção ao painel até que os pés tocassem-no (um ou ambos, simultaneamente ou não); 2) com sucesso, quando os lactentes tocaram os pés no painel elevando-o e, consequentemente, acionando o móbile. Além dessas categorias, foi analisada a duração de chute definido, que consistia no tempo cronometrado em segundos inferior de um ciclo do chute.

\section{Análise estatística}

Foi realizado o teste ANOVA three-way para medidas repetidas, seguido de Post-Hoc de Tukey para as variáveis dependentes: frequência total de chutes, frequência de contato dos pés no painel e duração do chute. Enquanto isso, para a variável dependente frequência de sucesso ao elevar o painel, foi utilizado o teste não paramétrico de Friedman. Ambos os testes foram aplicados com a finalidade de verificar a diferença entre os grupos, idades e condições experimentais, considerando um nível de significância de $5 \%$.

\section{Resultados}

Foram analisados, no total, 1.983 chutes, sendo 978 realizados pelos lactentes pré-termo e 1.005 realizados pelos lactentes a termo. 
Frequência total de chutes

Na variável frequência de chute, não foi constatada diferença significativa entre os lactentes pré-termo e a termo $[F(5,72)=1,117 ; p=0,447]$. Por outro lado, houve diferença significativa entre as idades $[\mathrm{F}(5,72)$ $=5,620 ; \mathrm{p}=0,0035]$, sendo que, aos 3 meses, ambos os grupos tiveram uma maior frequência de chutes durante as condições experimentais linha de base e peso, além de uma menor frequência de chutes na condição experimental peso, quando comparadas ao desempenho dos sujeitos com 4 meses de idade. Constatou-se também diferença entre as condições experimentais $[F(5,72)=9,760 ; p<0,001]$ linha de base e peso $(p=0,023)$ e peso e pós-peso $(p<0,001)$, sendo que em ambos os grupos e idades, os lactentes tiveram uma maior frequência de chutes nas condições experimentais linha de base e pós-peso, quando comparada ao obtido na condição peso (Gráfico 1).

\section{Frequência de contato dos pés no painel}

Não foi constatada diferença significativa entre os grupos $[\mathrm{F}(5,72)=3,887 ; \mathrm{p}=0,053]$ e as idades $[\mathrm{F}(5,72)$ $=1,041 ; \mathrm{p}=0,311]$ na variável frequência de contato. No entanto, foi constatada diferença significativa entre as condições experimentais $[F(5,72)=13,247$; $p<0,001]$, somente entre as condições experimentais linha de base e peso $(\mathrm{p}=0,002)$ e peso e pós-peso ( $p<0,001)$, indicando que em ambos os grupos e idades os lactentes tiveram uma maior frequência de contato dos pés no painel nas condições linha de base e pós-peso quando comparados a peso (Gráfico 2).

Frequência de sucesso ao elevar o painel

Na variável sucesso ao elevar o painel, constatou-se diferença entre os grupos aos 3 meses de idade $\left(\mathrm{x}^{2}{ }_{(1)}=4,341 ; \mathrm{p}=0,0013\right)$, lactentes a termo realizaram mais chutes com sucesso ao elevar o painel do que os lactentes pré-termo. Constatou-se também, diferença significativa entre linha de base e peso $(\mathrm{p}=$ $0,003)$, linha de base e pós-peso $(p=0,002)$ e peso e pós-peso $(\mathrm{p}=0,002)$ somente aos 3 meses de idade. De acordo com o Gráfico 3, verifica-se que, aos 3 meses, durante a condição experimental peso, ocorreu uma diminuição na frequência de sucesso para ambos os grupos; no entanto, na condição experimental

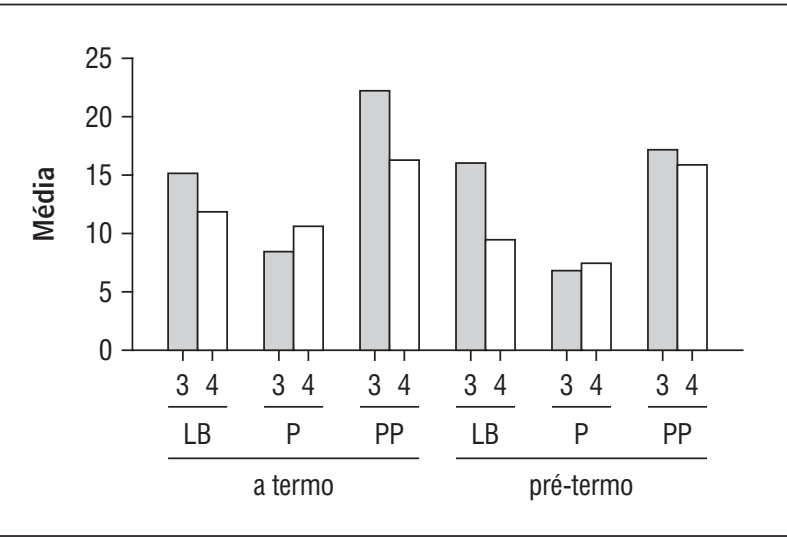

Gráfico 1 - Frequência total de chutes de lactentes pré-termo e a termo aos 3 e 4 meses de idade nas condições linha de base (LB), peso (P) e pós-peso (PP)

Fonte: Dados da pesquisa.

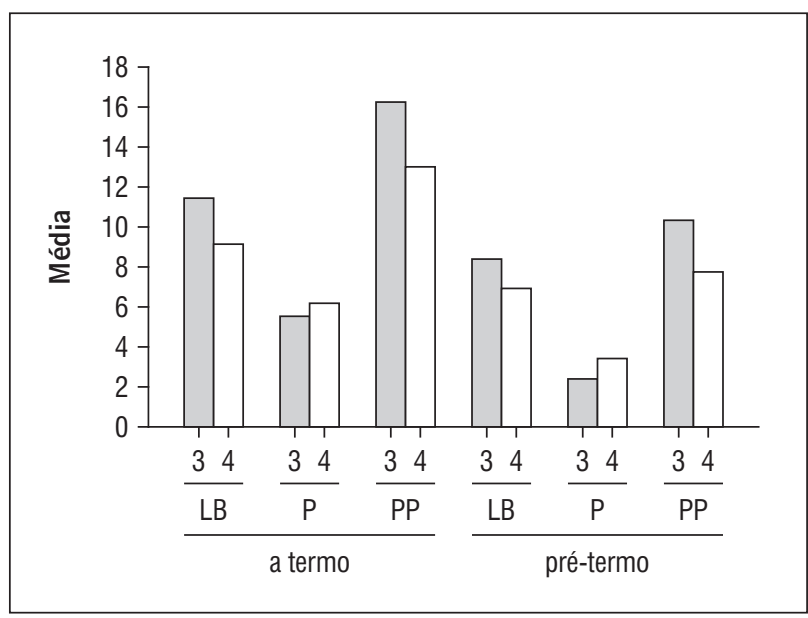

Gráfico 2 - Frequência de contato dos pés no painel de lactentes pré-termo e a termo aos 3 e 4 meses de idade nas condições linha de base (LB), peso (P) e pós-peso (PP)

Fonte: Dados da pesquisa.

pós-peso, houve um aumento na frequência de sucesso somente para os lactentes a termo quando comparada à linha de base.

\section{Duração do chute}

Observa-se que na variável duração do chute não foi verificada diferença significativa entre os grupos $[F(5,72)=0,268 ; p=0,634]$ e idades $[F(5,72)=0,382$; $\mathrm{p}=0,778$ ). Por outro lado, constatou-se diferença significativa entre as condições experimentais $[F(5,72)$ 
$=3,907 ; p=0,024]$ linha de base e peso $(p=0,023)$ e peso e pós-peso $(p=0,010)$, sendo que, durante a condição experimental peso, houve uma diminuição na duração do chute para ambos os grupos em ambas as idades, além de um aumento na duração do chute na condição experimental pós-peso (Gráfico 4).

\section{Discussão}

As variáveis contínuas, frequência total de chute, frequência de contato dos pés no painel e duração do chute, apresentam comportamento semelhante em ambos os grupos. Tal fato pode ser justificado pela idade gestacional avaliada dos lactentes pré-termo (média: 33,23 semanas) e pelo fato de todos os lactentes serem considerados saudáveis. Ou seja, os lactentes eram adequados para idade gestacional (AIG) e não apresentaram intercorrências peri e pós-parto. Isso pode ter contribuído para que os lactentes pré-termo fossem capazes de realizar a tarefa de forma semelhante aos lactentes a termo. De acordo com alguns autores, as diferenças no padrão de movimento de chute entre os grupos são menores aos 3 meses de idade em virtude das transformações neurais que ocorrem em tal período (26).

Em contrapartida, constatou-se diferença entre os grupos na variável frequência de sucesso ao elevar o painel aos 3 meses de idade, sendo observada uma menor frequência de sucesso no grupo pré-termo. Tal fato pode ser explicado pela fraqueza e hipotonia muscular apresentadas pelos lactentes pré-termo, que se tornam evidentes em tarefas mais complexas, nas quais temos a ação da gravidade associada ao peso do painel acentuando as características orgânicas (hipotonia e fraqueza muscular) presentes nos lactentes pré-termo (27). Aos 4 meses de idade, não se constatou diferença significativa, pois nesta idade o lactente pré-termo apresenta tônus e força musculares semelhantes aos do lactente a termo.

Outra variável em que observamos diferença entre as idades foi a variável frequência total de chutes. Os lactentes de ambos os grupos chutam com menor frequência aos 4 meses de idade nas condições LB e PP, nas quais não temos a restrição do peso. Acreditamos que tal fato pode ser explicado por meio do desenvolvimento motor; ou seja, entre os 3 e 6 meses de idade, os lactentes começam a alcançar $(28,29)$, explorando mais o meio com as mãos do que com os pés e, consequentemente, diminuem a

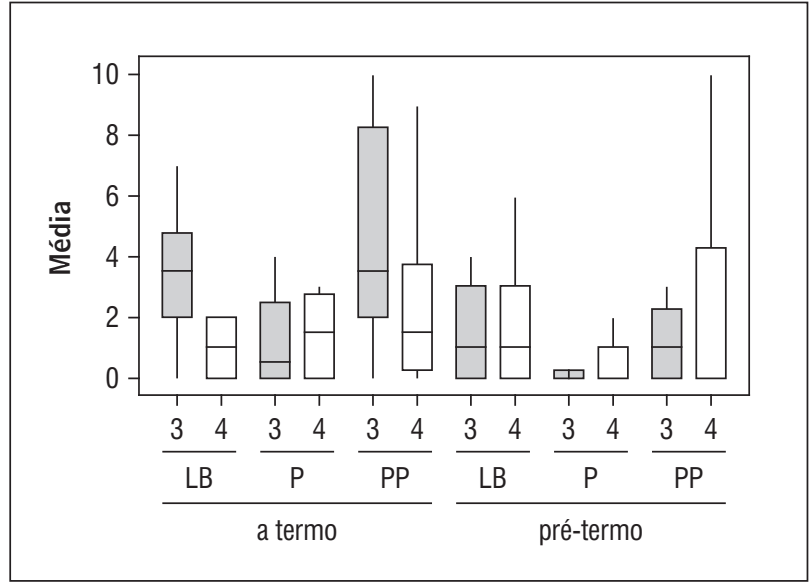

Gráfico 3 - Frequência de sucesso ao elevar o painel de lactentes pré-termo e a termo aos 3 e 4 meses de idade nas condições linha de base (LB), peso (P) e pós-peso (PP)

Fonte: Dados da pesquisa.

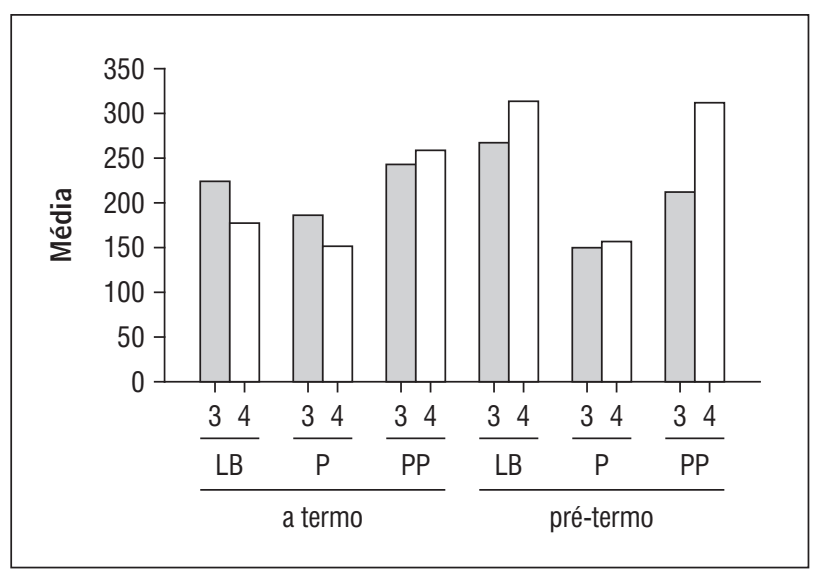

Gráfico 4 - Duração do chute de lactentes pré-termo e a termo aos 3 e 4 meses de idade nas condições linha de base (LB), peso (P) e pós-peso (PP)

Fonte: Dados da pesquisa.

frequência total de chutes. Entretanto, observa-se que, aos 4 meses de idade, durante a condição peso, os lactentes de ambos os grupos apresentaram maior frequência total de chutes, quando comparada à frequência dos lactentes com 3 meses de idade. Isso porque, nesta idade, eles apresentam menor força muscular, o que se torna mais evidente com o peso adicional, que, por sua vez, aumenta a dificuldade da tarefa, ocasionando a diminuição da frequência total de chutes. Essa redução na frequência total de chutes na condição experimental peso também poder ser atribuída à maturação do sistema nervoso central e 
ao ganho de força e coordenação muscular, ou seja, o cérebro do lactente passa por mudanças na maturação, plasticidade e memória-aprendizado (30). Dessa forma, uma vez que o lactente tenha realizado a mesma tarefa no mês anterior (terceiro mês) e sido treinado antes de iniciar a coleta, ele é capaz de utilizar a memória não declarativa para realizar o chute.

Essa diminuição na frequência durante a condição peso também é observada nas variáveis frequência de contato dos pés no painel, frequência de sucesso ao elevar o painel, além de uma diminuição na duração do chute. Isso ocorre em função do peso adicional que dificulta a tarefa, evidenciado pela diferença estatisticamente significativa entre as condições experimentais LB-P e P-PP. Sugerimos que essa variação ocorra em virtude da adaptação do lactente quanto ao peso. 0 lactente sofre mudança no comportamento em razão da alteração e ativação neural. Quando é colocada uma restrição no movimento, o cérebro, por meio da plasticidade, reage com alteração neural, refletindo em um novo comportamento $(18,31)$. No entanto, não foi observada diferença estatisticamente relevante entre as condições experimentais LB-PP, ou seja, os lactentes apresentaram comportamento semelhante antes e depois do peso adicional, evidenciando que esse acréscimo influenciou no chute momentaneamente. Desta forma, o peso alterou o comportamento das variáveis frequência total de chutes e frequência de contato dos pés nos painel apenas durante a condição peso.

Em contrapartida, em relação às variáveis frequência de sucesso ao elevar o painel e duração do chute, foi observada diferença estatisticamente significativa entre as condições experimentais LB-PP. Verifica-se um aumento na frequência de sucesso ao elevar o painel em ambos os grupos aos 4 meses de idade na condição PP, quando comparada à condição LB. Já aos 3 meses de idade foi observada uma elevação na frequência de sucesso na condição PP quando comparada ao LB apenas para os lactentes a termo. Isso ocorre em virtude da capacidade de percepção e adaptação do sistema somatossensorial (18). Uma vez adicionado o peso de $1 / 3$ da massa do membro, os mecanorreceptores detectam o peso adicional e utilizam essa informação extrínseca para selecionar um padrão adaptativo do chute, favorecendo a estabilidade do movimento e deixando-o mais direcionado à obtenção de maior sucesso.

Em relação à variável duração do chute, foi observado um aumento na duração do chute na condição experimental PP quando comparado à LB aos 3 e 4 meses de idade para ambos os grupos. Tal fato pode ser explicado em virtude do aumento na frequência de sucesso que consiste em manter o painel elevado; assim, quando o lactente faz uma extensão de joelho, mantém o painel elevado por um tempo prolongado, aumentando a duração do chute.

Dessa maneira, consideramos que as variáveis contínuas dos chutes de lactentes pré-termo se diferenciam dos lactentes de termo dependendo de suas características intrínsecas (restrições orgânica) e extrínsecas (ambiente e complexidade da tarefa). Uma vez que estas sejam similares, a diferença desaparece e os lactentes pré-termo e de termo apresentam o mesmo desempenho na tarefa de chutar.

\section{Referências}

1. Graziano RM, Leone CR. Problemas oftalmológicos mais freqüentes e desenvolvimento visual do pré-termo extremo. J Pediatr. 2005;81(1Supl):S95-100.

2. Miceli PJ, Goeke-Morey MC, Whitman TL, Kolberg KS, Miller-Loncar C, White RD. Brief report: birth status, medical complications, and social environment: individual differences in development of preterm, very low birth weight infants. J Pediatr Psychol. 2000; 25(5):353-8.

3. Halpern LF, Brand KL, Malone AF. Parenting stress in mothers of very-low-birth-weight (VLBW) and fullterm infants: a function of infant behavioral characteristics and child-rearing attitudes. J Pediatr Psychol. 2001;26(2):93-104.

4. Lekskulchai R, Cole J. Effect of a developmental program on motor, performance in infants born preterm. Aust J Physiother. 2001;47(3):169-76.

5. Linhares MBM. Estresse, resiliência e cuidados no desenvolvimento de neonatos de alto risco. In: Mendes EGM, Almeida A, Williams LCA. (Org.). Temas em Educação Especial. São Carlos: EDUFScar; 2004. p. $315-24$.

6. Weiss SJ. Haptic perception and the psychosocial functioning of preterm, low birth weight infants. Infant Behavior and Development. 2005;28(3):329-59.

7. Tavares EC, Rego MAS. Prematuridade e retardo de crescimento. In: Lopez FA, Campos D Jr. (Org.). Tratado de Pediatria. Barueri: Manole; 2007. p. 1315-23. 
8. Ayache MG, Mariani CN. Considerações sobre o desenvolvimento motor do prematuro. Temas sobre Desenvolvimento. 2003;12(71):5-9.

9. Piek JP. Is a quantitative approach useful in the comparison of spontaneous movements in full-term and preterm infants? Hum Mov Sci. 2001;20(4-5):717-36.

10. Fallang B, Saugstad AD, Haddres-Algra M. Postural adjustments in preterm infants at 4 and 6 months post-term during voluntary reaching in supine position. Pediatr Res. 2003;54(6):826-33.

11. Morison SJ, Holsti L, Grunau RE, Whitfield MF, Oberlander TF, Chan HWP, et al. Are there developmentally distinct motor indicators of pain in preterm infants? Early Hum Dev. 2003;72(2):131-46.

12. Thelen E, Bradshaw G, Ward JA. Spontaneous kicking in month-old infants: manifestation of a human central locomotor program. Behav Neural Biol. 1981; 32(1):45-53.

13. Thelen E, Fisher DM. The organization of spontaneous leg movements in newborn infants. J Mot Behav. 1983;15(4):353-77.

14. Piek JP. A quantitative analysis of spontaneous kicking in two-month-old infants. Hum Mov Sci. 1996;15(5): 707-26.

15. Magill RA. Aprendizagem motora: conceito e aplicações. São Paulo: Edgard Blucher; 2000.

16. Vaal J, van Soest AJ, Hopkins B, Sie LTL. Spontaneous leg movements in infants with and without periventricular leukomalacia: effects of unilateral weighting. Behav Brain Res. 2002;129(1-2):83-92.

17. Piek JP, Gasson N. Spontaneous kicking in full-term and preterm infants: are there leg asymmetries? Hum Mov Sci. 1999;18(2-3):377-95.

18. Thelen E, Skala KD, Kelso JA. The dynamic nature of early coordination: evidence from bilateral leg movements in young infants. Dev Psychol. 1987;23(2): 179-86.

19. Vaal J, Soest AJ, Hopkins B, Knaap MS. Development of spontaneous leg movements in infants with and without periventricular leukomalacia. Exp Brain Res. 2000;135(1):94-105.

20. Ulrich BD. Sensitivity of infants with and without Down syndrome to intrinsic dynamics. Res Q Exerc Sport. 1997;68(1):10-9.
21. Bussab WO, Moretin PA. Estatística básica. São Paulo: Saraiva; 2002.

22. Schneider K, Zernicke RF. Mass, centre of mass, and moment of inertia estimates for infant limb segments. J Biomech. 1992;25(2):145-8.

23. Landgraf JF, Tudella E. Efeitos do peso externo nos chutes espontâneos de lactentes nos primeiros dois meses de vida. Rev Bras Fisioter. 2008;12(6):468-75. doi.org/10.1590/S1413-35552008005000009

24. Jeng SF, Chen LC, Yau Kit. Kinematic analysis of kicking movements in preterm infants with very low birth weight and full-term infants. Phys Ther. 2002;82(2): 148-59.

25. Pretchl HFR, Beintema DJ. The neurological examination of the full-term newborn infant. Clin Dev Med. 1964;12:1-73.

26. Geerdink JJ, Hopkins B, Beek WJ, Heriza CB. The organization of leg movements in preterm and full-term infants after term age. Dev Psychobiol. 1996;29(4): 335-51.

27. Wilson SL, Cradock MM. Review: accounting for prematurity in Developmental Assessment and the Use of Age-Adjusted Scores. J Pediatr Psychol. 2004;29(8): 641-9.

28. Berthier NE, Clifton RK, MacCall DD, Robin DJ. Proximodistal structure of early reaching in human infants. Exp Brain Res. 1999;127(3):259-69.

29. Thelen E, Corbetta D, Kamm K, Spencer JP, Schneider $\mathrm{K}$, Zernicke RF. The transition to reaching: mapping intention and intrinsic dynamics. Child Dev. 1993; 64(4):1058-98.

30. Cardoso SH. Memória o que é e como melhorá-la. [acesso 18 jun. 2011]. Disponível em: http://www. cerebromente.org.br/n01/memo/memoria.htm.

31. Schmidt R. Aprendizagem e perfomance motora. São Paulo: Artmed; 2001.
Recebido: 07/ 12/2010

Received: 12/ 07/2010

Aprovado 01/08/2011

Approved: 08/01/2011 